\title{
Reproductive Dysfunctions in Males with Type 2 Diabetes Mellitus: An Updated Review
}

Authors:

Disclosure:

Received:

Accepted:

Keywords:

Citation:
*Sayantan Ray, ${ }^{1}$ Subhodip Pramanik²

1. Department of Endocrinology and Metabolism, Medica Superspeciality Hospital, Kolkata, India

2. Department of Endocrinology and Metabolism, Neotia Getwel Healthcare Centre, Siliguri, India

*Correspondence to sayantan.ray30@gmail.com

The authors have declared no conflicts of interest.

15.07.20

24.09.20

Erectile dysfunction (ED), hypogonadism, infertility, obesity, phosphodiesterase inhibitors, testosterone therapy, Type 2 diabetes mellitus (T2DM).

EMJ Diabet. 2020;8[1]:79-89.

\section{Abstract}

Deterioration in reproductive functions is one of the most serious complications of Type 2 diabetes mellitus (T2DM). Neuropathy, angiopathy, oxidative stress, and psychological deviation are the important causative factors in developing reproductive dysfunctions in diabetes. In males, the principal complications are erectile dysfunction (ED), ejaculatory disorders, and functional hypogonadism. Low serum testosterone is frequently observed in males with T2DM but the neuroendocrine pathophysiology is yet to be defined; this reduction in testosterone levels decreases libido. Evaluation of testosterone levels of male diabetic patients with hypogonadism symptoms is recommended. Hypogonadal males with diabetes might benefit from testosterone replacement therapy. However, there is a need for adequately powered long-term studies in this context. Impairment in sexual function is a common complication in males with diabetes. The pathophysiology of sexual dysfunction in diabetes is multifactorial. Males with diabetes have a >3-fold increase in the risk of ED compared to their nondiabetic counterparts. Phosphodiesterase type 5 inhibitors should be considered as first-line therapy in males with T2DM and ED. Nearly $50 \%$ of male diabetic patients presented some degree of subfertility or infertility. Alterations in sperm parameters and hormone levels can contribute to diabetes-related male infertility. Endocrinologists, diabetologists, and physicians should address sexual complaints of their patients since these problems can significantly impair their quality of life.

\section{INTRODUCTION}

Type 2 diabetes mellitus (T2DM) is a major health concern worldwide. The Southeast Asian region has the second largest number of people with diabetes in the world. The prevalence of T2DM is six times greater in males of Southeast Asian origin.' T2DM can affect multiple physiological systems, including the reproductive system. T2DM causes sexual dysfunctions in males through autonomic neuropathy and endothelial dysfunction. ${ }^{2}$ The most frequently reported sexual dysfunction is erectile dysfunction (ED), the prevalence of which among males with diabetes varies from 35 to $75 \% .^{3}$ In males, 
strong evidence linking low testosterone levels to T2DM and metabolic syndrome (MetS) is there. Up to $40 \%$ of males with T2DM and MetS have hypogonadism. ${ }^{4}$ Hypogonadal males with diabetes have a higher risk for cardiovascular (CV) mortality than eugonadal males. ${ }^{5}$ Altogether, these abnormalities may lead to a decrease in male fertility. However, a comprehensive overview regarding pathophysiology, consequences, evaluation, and treatment of reproductive complications of T2DM in males is lacking. Therefore, this paper aims to provide an updated review of the various aspects of this complication.

\section{IMPACT OF DIABETES ON MALE SEXUAL AND REPRODUCTIVE FUNCTIONS}

The pathophysiology of reproductive dysfunction in diabetes is multifactorial. The longer the duration of diabetes and the older the patient, the more likely that they are to have sexual dysfunction. ${ }^{6}$ Comorbidities including hypertension, dyslipidaemia, CV disease (CVD), or other endocrine dysfunctions, and their treatments, can further expedite sexual and reproductive impairment. ${ }^{2}$ In males with diabetes, low testosterone levels are more common and can cause reduced libido and ED. Finally, the complex role of psychological issues in diabetes contributes to impaired sexual function. The following sections will detail the issues related to hypogonadism and sexual dysfunction in males with diabetes.

\section{Hypogonadism in Males with Diabetes}

In males with T2DM, subnormal free testosterone concentrations in association with inappropriately low luteinizing hormone (LH) and follicle-stimulating hormone concentrations were first described by Dhindsa et al. ${ }^{7}$ These abnormalities were not dependent on the severity of hyperglycaemia. The reported prevalence of hypogonadotropic hypogonadism $(\mathrm{HH})$ in males with T2DM is $30-40 \% .^{8-10}$ Chandel et al. ${ }^{11}$ found that younger males with T2DM also have a similarly high prevalence of $\mathrm{HH}$. In all the studies, testosterone levels were inversely correlated to $\mathrm{BMI}$ and insulin resistance (IR). ${ }^{12}$ A high prevalence of symptoms suggestive of hypogonadism has been found in males with
T2DM with low testosterone concentrations. ${ }^{8}$ Given the inverse relationship between BMI and testosterone levels in T2DM, $\mathrm{HH}$ is most likely related to IR. 7,9

\section{Pathophysiological mechanisms underlying $\mathrm{HH}$}

The causative mechanism of diabetesinduced $\mathrm{HH}$ remains to be elucidated but is probably multifactorial. Several factors may be associated with the pathophysiology, including age, IR, obstructive sleep apnoea (OSA), and visceral obesity. ${ }^{10,13}$

It has been theorised that increased oestrogen production due to aromatase activity in the obese may potentially suppress the hypothalamic gonadotropin-releasing hormone $(\mathrm{GnRH})$ secretion. However, Dhindsa et al. ${ }^{14}$ have shown that total and free oestradiol levels in males with $\mathrm{HH}$ are considerably lower than in those without $\mathrm{HH}$. So, it appears that the low testosterone levels in diabetes-related $\mathrm{HH}$ are not the consequence of oestradiol-induced suppression of the hypothalamic-pituitary-gonadal (HPG) axis.

Obesity and T2DM are associated with decreased insulin signalling in the central nervous system. While the site responsible for hypogonadotropism in neuronal IR is unknown, it is apparent that insulin action and responsiveness in the brain are essential for the functional integrity of the HPG axis. $^{5}$

Leptin appears to serve as a signal of energy reserves to regulate the HPG axis with nutritional status. Leptin resistance in the hypothalamus or some other neurons may be related to the hypogonadotropism found in diabesity. 5,15 Kisspeptin and the presence of kisspeptin receptors on the $\mathrm{GnRH}$ neurons are required for the $\mathrm{GnRH}$ release. Kisspeptin infusion raises $\mathrm{LH}$ and testosterone levels in males with T2DM and $\mathrm{HH}^{16,17}$ Both leptin and insulin receptors are expressed in kisspeptin neurons. Hence, the hypothalamic defect in male T2DM patients with $\mathrm{HH}$ is either at kisspeptin level or proximal to it.

TNFa and IL-1B have been demonstrated to suppress hypothalamic $\mathrm{GnRH}$ release in experimental animals. ${ }^{18}$ Thus, it is relevant that C-reactive protein concentrations are significantly increased in males with $\mathrm{HH}$ and $\mathrm{T}^{2} \mathrm{DM} .{ }^{19}$ It is, therefore, possible that inflammatory mediators may be responsible for the suppression of 
the HPG axis and consequently $\mathrm{HH}$ in T2DM. The presence of inflammation may also lead to IR. ${ }^{20}$

In summary, various interconnected mechanisms are underlying $\mathrm{HH}$ in males with T2DM (Figure 1). The absence of an increase in gonadotropin levels suggests that the primary defect is at the HP level. ${ }^{5}$

\section{Consequences of hypogonadism in Type 2 diabetes mellitus}

\section{Sexual dysfunction}

A high prevalence of low libido (64\%), ED (74\%), and fatigue (63\%) has been found in hypogonadal males with $\mathrm{T}^{2 D M}{ }^{8}$ It is often difficult to establish whether the aetiology of symptoms is hypogonadism or any other comorbidity. Nevertheless, the prevalence of sexual symptoms is higher in males with $\mathrm{HH}$ than eugonadal males. ${ }^{21}$ In hypogonadal males with T2DM, trials of testosterone replacement therapy (TRT) have also shown an improvement in libido. ${ }^{21,22}$

\section{Insulin resistance}

Dhindsa et al. ${ }^{21}$ have shown that males with T2DM and $\mathrm{HH}$ are less insulin sensitive than those without $\mathrm{HH}$ and that TRT increases insulin sensitivity.

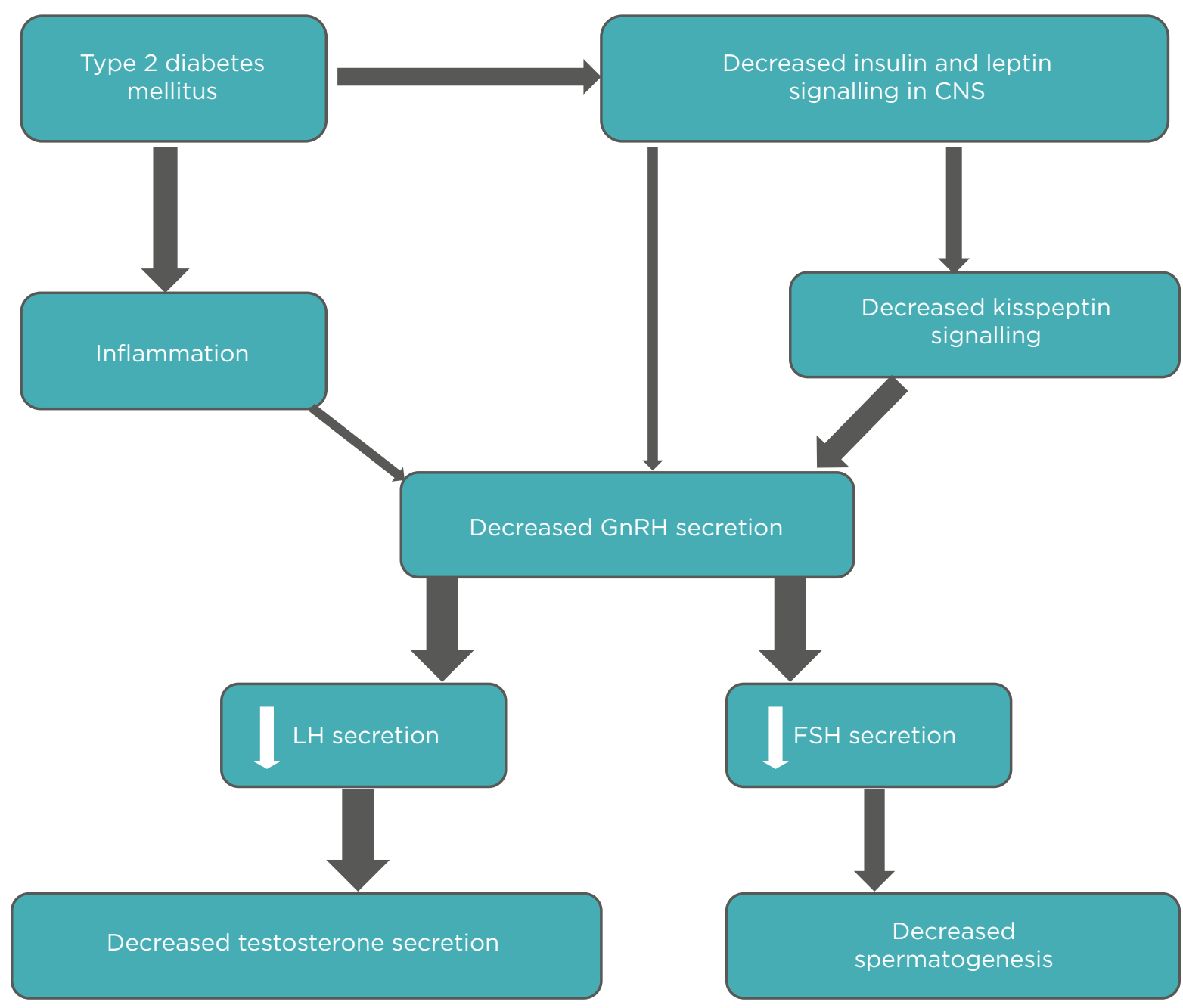

Figure 1: Interplay of different factors in diabetes-associated hypogonadotropic hypogonadism.

The thickness of arrows is proportional to the strength of available evidence.

CNS: central nervous system; FSH: follicle-stimulating hormone; GnRH: gonadotropin-releasing hormone; LH: luteinizing hormone.

Adapted from Dhindsa et al. ${ }^{5}$ 
However, two trials did not show a change in HOMA-IR after testosterone replacement in hypogonadal males with T2DM. ${ }^{23,24}$ Since TRT increases muscle mass, TRT may have a more notable effect on muscle glucose uptake rather than HOMA-IR. Thus, testosterone mediated insulin sensitisation is not an immediate effect and may be related to changes in body composition. The improvement in insulin sensitivity following TRT was also associated with suppression of inflammatory mediators. ${ }^{5}$ Whether they are the direct actions of testosterone remain to be determined.

\section{Cardiovascular disease}

Low circulating testosterone levels lead to greater visceral adiposity and increased cardiometabolic risk. $^{4}$ The inverse relationship of mortality with endogenous testosterone levels has been observed in males with diabetes. ${ }^{25}$ Hypogonadism has been associated both with CVD risk factors in males with $\mathrm{T} 2 \mathrm{DM},{ }^{26}$ and an increased risk of myocardial infarction and increased CV mortality. ${ }^{27,28}$ However, no randomised control trials (RCT) have been conducted to address whether TRT changes CV outcomes in males.

\section{Anaemia}

Males with T2DM and $\mathrm{HH}$ have a lower haematocrit than those with normal testosterone concentrations. The prevalence of normocytic normochromic anaemia in such patients is higher in comparison to eugonadal males. ${ }^{19}$ However, it remains undetermined whether the association of anaemia with hypogonadism in males with T2DM is causative.

\section{Bone density}

Hypogonadism is associated with a decline in bone mineral density and an increase in fracture rate. $^{29}$ In epidemiological studies, oestradiol concentrations correlate more strongly with bone mineral density than testosterone concentrations in males. However, testosterone appears to be an independent predictor of cortical bone density. ${ }^{30}$ Free testosterone levels have shown a positive association with bone density in arms, ribs, and lumbar spine in males with T2DM. ${ }^{31}$ No data are available on the fracture rates in males with T2DM and $\mathrm{HH}^{12}$

\section{Evaluation and treatment of hypogonadism}

\section{Evaluation of hypogonadal symptoms}

Low testosterone concentrations are associated with symptoms such as fatigue, lack of libido, and ED. Importantly, patients may also slide slowly into this clinical state without any obvious symptoms. ${ }^{12}$ The high prevalence of low testosterone levels in T2DM justifies screening for $\mathrm{HH}$ in every patient with T2DM; however, biochemical testing is recommended in males with diabetes who are symptomatic. In 2016, the American Academy of Clinical Endocrinologists (AACE) recommended screening for hypogonadism in all males with T2DM. ${ }^{32}$ In contrast, the 2018 Endocrine Society (ES) guidelines continue to advocate against testosterone screening but do acknowledge the high prevalence of hypogonadism in T2DM.33 The recent reclassification of hypogonadism by the ES refers to T2DM-related hypogonadism as "functional". The American Diabetes Association (ADA) in 2018 acknowledged the high prevalence of $\mathrm{HH}$ in T2DM and recommended testosterone measurement in males with symptomatic hypogonadism. ${ }^{34}$ The ES focusses on correct diagnosis and proper assay techniques, though there is little that the clinician can do about the assay method. Ideally, free testosterone should be measured in the morning (fasting) by accurate methodology, preferably equilibrium dialysis/mass spectrometry. Subnormal free testosterone should be confirmed at least once. A male with confirmed hypogonadism should have LH concentrations checked. T2DM and obesity are associated with reduced sex hormone-binding globulin (SHBG) concentrations. Thus, a physiological lowering of total testosterone concentrations is expected in obese males with T2DM. Therefore, free or bioavailable testosterone measurement is necessary to assess the gonadal status in these patients.

OSA is very common in people with T2DM and obesity. However, OSA per se is not the main contributor to the decline of testosterone concentrations. $^{5}$ Prostate-specific antigen concentrations are found to be lower in hypogonadal than in eugonadal males with diabetes. ${ }^{35}$ 


\section{Treatment of hypogonadism}

Studies with TRT suggest remarkable benefits in sexual function, quality of life, lean muscle mass, and bone density. ${ }^{4}$ ADA recommends considering TRT in cases for which evidence indicates likely improvements, but it does not consider any further metabolic role for TRT. ${ }^{34}$ The ES guideline recommends against TRT for glycaemic control but does endorse TRT in males with ED and low sexual desire. ${ }^{33}$ A complete discussion of risks and benefits between the patient and physician should precede a trial of TRT. Monitoring the effects of testosterone should be done as per available guidelines, such as those recommended by the ES. Of note, the hypogonadism in diabetes is associated with reduced responsiveness to testosterone. ${ }^{5}$

Weight loss associated increase in testosterone concentrations is likely mediated by the restoration of neuronal leptin and insulin sensitivity. Many studies have also shown that weight loss increases total testosterone and SHBG concentrations. ${ }^{36}$ Nevertheless, the results of lifestyle intervention as the sole therapy for hypogonadism in T2DM are discouraging.

\section{Metabolic effects of testosterone therapy in males with Type 2 diabetes mellitus}

The current evidence on the metabolic effects of TRT in men with T2DM and MetS is reviewed. ${ }^{4}$ RCT of TRT suggest considerable benefits in terms of improved IR, decreased fat mass, increased lean muscle mass, and reductions of inflammatory markers. Altogether, these might be expected to translate into reduced long-term CV risk. However, critical evaluation of clinical trials has been complicated by various factors. Since all males with T2DM are routinely prescribed a statin irrespective of lipid level, it would be very difficult to conduct RCT of TRT in dyslipidaemia. Several longitudinal and observational studies also suggest long-term persistent improvements in metabolic parameters. However, presently TRT is not considered a mainstream intervention in standard diabetes practice. Although no studies have demonstrated an association between TRT and increased risk of major adverse CV events (MACE), some effects of testosterone might enhance CV risk. ${ }^{37}$ The ongoing TRAVERSE study $^{38}$ is likely to clarify the testosterone effects on CVD.

\section{Sexual Dysfunction in Males with Diabetes}

Sexual dysfunction is a distressing complication in males with diabetes. It negatively impacts the quality of life and it is often an early clinical indication of endothelial dysfunction, thus predicting future CVD.

\section{Erectile dysfunction}

The prevalence of ED depends on the age of the patient, duration of diabetes, and presence of other comorbid conditions. ${ }^{39}$ According to a recent meta-analysis, the prevalence of ED in diabetes was $52.5 \%$, and it increased with the duration of diabetes. ${ }^{40}$

\section{Pathophysiology}

Erection is a vascular process that is initiated by the autonomic nervous system and maintained by endothelial cell-derived nitric oxide (NO) and endothelium-derived hyperpolarising factor (EDHF), which are responsible for smoothmuscle relaxation in the corpus cavernosum. Hyperglycaemia disrupts this physiology in multiple ways.

$>$ Endothelial dysfunction: failure of endothelium-derived $\mathrm{NO}$ and EDHF occurs much before significant autonomic neuropathy. ${ }^{41}$ Thus, there is difficulty in maintaining an erection.

$>$ Low testosterone: this leads to a reduction in smooth muscle sensitivity to vasodilators and structural abnormalities in the erectile tissue. ${ }^{42}$

> Large vessel disease: narrowing of large vessels supplying corpora cavernosa can lead to vascular impotence.

> Autonomic neuropathy: erection is initiated by cholinergic and non-cholinergic nonadrenergic neurotransmitters from the parasympathetic nervous system. They initiate the relaxation of the cavernosal smooth muscle. Autonomic neuropathy impairs this response.

> Drugs: $\beta$-blockers, thiazide diuretics, tricyclic antidepressants, and spironolactone are the major culprits. ${ }^{43}$

> Others: penile structural diseases, such phimosis and Peyronie's disease, or recurrent mycotic balanitis may have a contributory effect on ED in males with diabetes. ${ }^{44}$ 


\section{$E D$ is a harbinger of future cardiovascular dysfunction}

Numerous investigators reported ED as a marker of potential future CV events. ${ }^{45}$ Yamada et al. ${ }^{46}$ found a significant association of ED with allcause mortality and CV events in a meta-analysis. However, ED often precedes overt CVD, which clinicians often pay less attention to.

\section{Clinical assessment}

Most patients hesitate to initiate discussion about their sexual dysfunction. However, once initiated, ice can be broken easily. Clinicians need to first ascertain the degree of ED, presence or loss of libido, association with premature ejaculation (PME) or retrograde ejaculation, and presence/ absence of nocturnal penile tumescence. Several validated questionnaires can be used for the diagnosis of ED. The International Index of Erectile Function (IIEF) ${ }^{47}$ is currently the most widely used questionnaire. Situational or psychogenic ED can be easily diagnosed by a simple stamp paper test or RigiScan ${ }^{\circledR}$ (Gesiva Medical, Eden Prairie, Minnesota, USA), a device that monitors for the spontaneous nocturnal penile tumescence during rapid eye movement (REM) sleep. An overnight recording is done in a single room with quiet surroundings. The normal response is defined as $>70 \%$ rigidity and $3-4$ erections lasting $>10$ min. ${ }^{48}$

\section{Investigations}

Every patient should be evaluated for glycaemic control, CV, renal, retinal, and neurological status. Hypogonadism should be excluded by measuring serum testosterone levels. The pharmacological test with intracavernosal injections of papaverine and Doppler evaluation of the pre- and postapplication blood flow are often needed to exclude vascular cause of ED.

\section{Treatment}

> General advice

Optimal glycaemic control should be achieved in all patients. The use of statin and ramipril has also been reported to reduce ED. ${ }^{49,50}$ Most patients with situational ED improve with simple tips and advice, whereas few couples need psychosexual counsellors.

$>$ Oral agents

Phosphodiesterase type 5 (PDE5) inhibitors are first-line therapies (Table 1). They inhibit the breakdown of cyclic guanosine monophosphate (cGMP), which acts as a second messenger for NO-induced smooth-muscle relaxation. In males with T2DM, the efficacy rates have been reported to be between $50 \%$ and $55 \%$ and the average dose required is higher than the general population. ${ }^{48}$ Recent evidence suggests therapy with PDE5 inhibitors improves insulin sensitivity. ${ }^{51}$ Clinicians have generally shown a preference for tadalafil over sildenafil due to the long half-life, ease of administration, and favourable safety profile. Nonresponders are diagnosed when satisfactory sexual function is not achieved despite using an agent at least eight times in the highest recommended or tolerated dose.

\section{> Injectables}

Alprostadil is supplied in a self-injection pen device. In a 6-month self-injection study involving 683 males, the participants reported achieving satisfactory sexual activity after 94\% of the injections. ${ }^{52}$ Prolonged erections, priapism, penile fibrotic complications, and haematoma or ecchymosis were the most common side effects. Despite its high efficacy, the discontinuation rate was very high. ${ }^{53}$

$>$ Surgical options

Surgical options for ED are either correction of penile structural and vascular disease or insertion of a penile prosthesis. A penile prosthesis is best reserved for those in whom conventional treatments have failed and who are keen to resume full sexual activity. ${ }^{54}$

\section{Ejaculatory dysfunction}

Ejaculatory disorders are a heterogeneous group of disorders that are very common in people with diabetes and include premature and retrograde ejaculation.

\section{Premature ejaculation}

PME is defined as ejaculation that always or nearly always occurs before or within approximately 1 minute of vaginal penetration or inability to delay ejaculation on all or nearly all vaginal penetrations that lead to negative personal consequences.

In diabetes, psychological factors, such as depression, impaired self-body image, and performance anxiety, may play a role in the development of PME. Although a smaller number of couples seek medical attention for PME, it may be highly distressing in some instances. ${ }^{55}$ 
Table 1: Comparison of available PDE5 inhibitors.

\begin{tabular}{|c|c|c|c|c|}
\hline & Sildenafil & Tadalafil & Vardenafil & Avanafil \\
\hline $\mathrm{T}_{\max }$ & 1 hour & 2 hours & 1.5 hours & 0.75 hour \\
\hline$V_{d}$ & $105 \mathrm{~L}$ & $63 \mathrm{~L}$ & 208 L & -- \\
\hline Protein binding & $96 \%$ & $94 \%$ & $95 \%$ & $99 \%$ \\
\hline Major Metabolism & CYP3A4 & CYP3A4 & CYP3A4 & CYP3A4 \\
\hline Half-life & 4 hours & 17.5 hours & 4-6 hours & 5 hours \\
\hline $\begin{array}{l}\text { Ingestion with high-fat } \\
\text { meals }\end{array}$ & $\begin{array}{l}\downarrow C_{\max } 29 \% \\
\uparrow T_{\max } \text { by } 1 \text { hour } \\
\end{array}$ & Not affected & $\downarrow C_{\max } 35 \%$ & $\begin{array}{l}\downarrow C_{\max } 24-39 \\
\uparrow T_{\max } \text { by } 1.12-1.25 \text { hours }\end{array}$ \\
\hline Usual dosage & 25-100 mg/day & $\begin{array}{l}\text { 5-20 mg/day (as } \\
\text { needed); } \\
2.5-5 \mathrm{mg} / \text { day once } \\
\text { daily }\end{array}$ & 5-20 mg/day & 50-200 mg/day \\
\hline Administration time & $\begin{array}{l}1 \text { hour before sexual } \\
\text { activity }\end{array}$ & $\begin{array}{l}\text { At least } 0.5 \text { hours } \\
\text { before sexual activity }\end{array}$ & $\begin{array}{l}1 \text { hour before sexual } \\
\text { activity }\end{array}$ & $\begin{array}{l}0.5 \text { hours before } \\
\text { sexual activity }\end{array}$ \\
\hline Time frame of efficacy & $0.5-4$ hours post dose & $\begin{array}{l}\text { Up to } 36 \text { hours post } \\
\text { dose }\end{array}$ & -- & $\begin{array}{l}\text { As early as } 0.25 \text { hours } \\
\text { post dose }\end{array}$ \\
\hline $\begin{array}{l}\text { Common adverse } \\
\text { reactions }\end{array}$ & $\begin{array}{l}\text { Headache, flushing, } \\
\text { dyspepsia, nasal } \\
\text { congestion, } \\
\text { nasopharyngitis, visual } \\
\text { abnormalities }\end{array}$ & $\begin{array}{l}\text { Headache, flushing, } \\
\text { dyspepsia, nasal } \\
\text { congestion, } \\
\text { nasopharyngitis, back } \\
\text { pain, myalgia }\end{array}$ & $\begin{array}{l}\text { Headache, flushing, } \\
\text { dyspepsia, nasal } \\
\text { congestion, } \\
\text { nasopharyngitis, visual } \\
\text { abnormalities }\end{array}$ & $\begin{array}{l}\text { Headache, flushing, } \\
\text { dyspepsia, nasal } \\
\text { congestion, } \\
\text { nasopharyngitis }\end{array}$ \\
\hline $\begin{array}{l}\text { Time required } \\
\text { from last dose to } \\
\text { administration of a } \\
\text { nitrate }\end{array}$ & 24 hours & 48 hours & 24 hours & 12 hours \\
\hline
\end{tabular}

$\mathrm{C}_{\text {max }}$ : maximum concentration; CYP3A4: cytochrome P450 3A4; PDE5: phosphodiesterase 5; $T_{\text {max }}$ : time to reach maximum concentration; $V_{d}$ : volume of distribution.

\section{Retrograde ejaculation}

Retrograde ejaculation as a complication of diabetes is unappreciated and under-recognised. The exact prevalence of retrograde ejaculation in diabetic patients is unknown. It is the propulsion of seminal fluid from the posterior urethra retrograde to the bladder through a relaxed internal vesical sphincter. It is considered to be a feature of diabetic autonomic neuropathic manifestation, ${ }^{56}$ and presents with infertility. Spermatozoa can be retrieved from centrifuged urine after a sexual act. Medical treatment with 
imipramine and pseudoephedrine have been tried, but with limited efficacy. Alternatively, spermatozoa retrieval from post-ejaculatory urine can be a good alternative to couples presenting with infertility.

\section{DIABETES MELLITUS AND MALE INFERTILITY}

There will be a growing number of males of reproductive age with diabetes, as there is an increase in the number of adolescent males with T2DM. ${ }^{57}$ The view that diabetes has insignificant effects on male reproductive function has been questioned by current data. ${ }^{58}$

Diabetes can have deleterious effects on male reproductive health, which can lead to increased infertility. Various studies have reported diverse pathologies and consequent reproductive defects. Selected mechanisms are summarised in Figure 2.

\section{Modified Semen Parameters}

Modified semen parameters can contribute to male infertility in diabetes. Diabetes induces subtle molecular alterations affecting sperm quality and function. Semen analysis revealed a significant decline in sperm motility, including the number of rapid progressive cells in males with diabetes. ${ }^{59}$ Another study revealed decreased sperm motility and increased abnormal sperm morphology of male diabetic partners. ${ }^{60}$ Diabetic patients may have a decrease in semen volume, sperm count, and motility along with increases in seminal glucose levels and decreases in zinc concentration.61,62 Mitochondrial dysfunctions may also account for the deteriorated sperm parameters. ${ }^{2}$ Advanced glycation end products in seminal plasma could be a major contributor to oxidative stress and therefore sperm nuclear DNA damage. ${ }^{63}$ Larger studies are necessary to confirm many of these findings.

\section{Hyperglycaemia-Related Male Infertility}

To date, several studies have addressed the issue of diabetes-induced male infertility but comprehensive evidence as to how hyperglycaemia impairs male fertility is absent. The hyperglycaemia may cause testicular dysfunction by disrupting both steroidogenesis and spermatogenesis.

Additionally,

hyperglycaemia impairs male reproductive function through increased oxidative stress. ${ }^{64}$ Diabetes-related testicular dysfunction includes decreased spermatogenesis due to an increased rate of germ cell apoptosis, poor sperm reproductive parameters, and reduced testosterone synthesis, resulting in infertility. ${ }^{58}$ Hyperglycaemia should be investigated in more detail to fully understand the impact on male reproductive health.

\section{Treatment of Diabetes-Induced Male Infertility}

Currently, a specific treatment to improve reproductive dysfunctions in T2DM patients is not available. Hormonal approaches with growth hormone and human chorionic gonadotropin were tried in diabetes-induced infertility. ${ }^{65}$ While the effects of hyperglycaemia reduction by the use of insulin and few oral drugs have been reported, more clinical trials providing high-quality evidence on the positive effects on male reproduction are necessary. ${ }^{64}$ Antioxidant therapy remains highly debated despite the reported improvement of sperm quality. ${ }^{66}$

\section{CONCLUDING REMARKS}

Currently, T2DM is growing rapidly among adolescent males, resulting in a significant increase in the prevalence of reproductive dysfunction among young males. Males with T2DM may demonstrate hypogonadism, ED, and ejaculatory dysfunction, leading to infertility. The low testosterone level seen in males with T2DM is associated with increased comorbidity and mortality. The available evidence suggests that males with T2DM, MetS, and properly diagnosed $\mathrm{HH}$ are likely to benefit from TRT. Results of ongoing trials can provide a more unified perspective on testosterone. All adult males with T2DM should be screened for ED with a sexual function history and PDE5 inhibitors should be offered to males who would like to undergo treatment for ED. Psychosexual therapy can be helpful when sex cannot return to normal for the patient. Clinicians should not only educate their patients about the possible impact of diabetes on male reproductive health, but also address their sexual complaints. 
Type 2 diabetes mellitus

(lifestyle factors; insulin resistance)

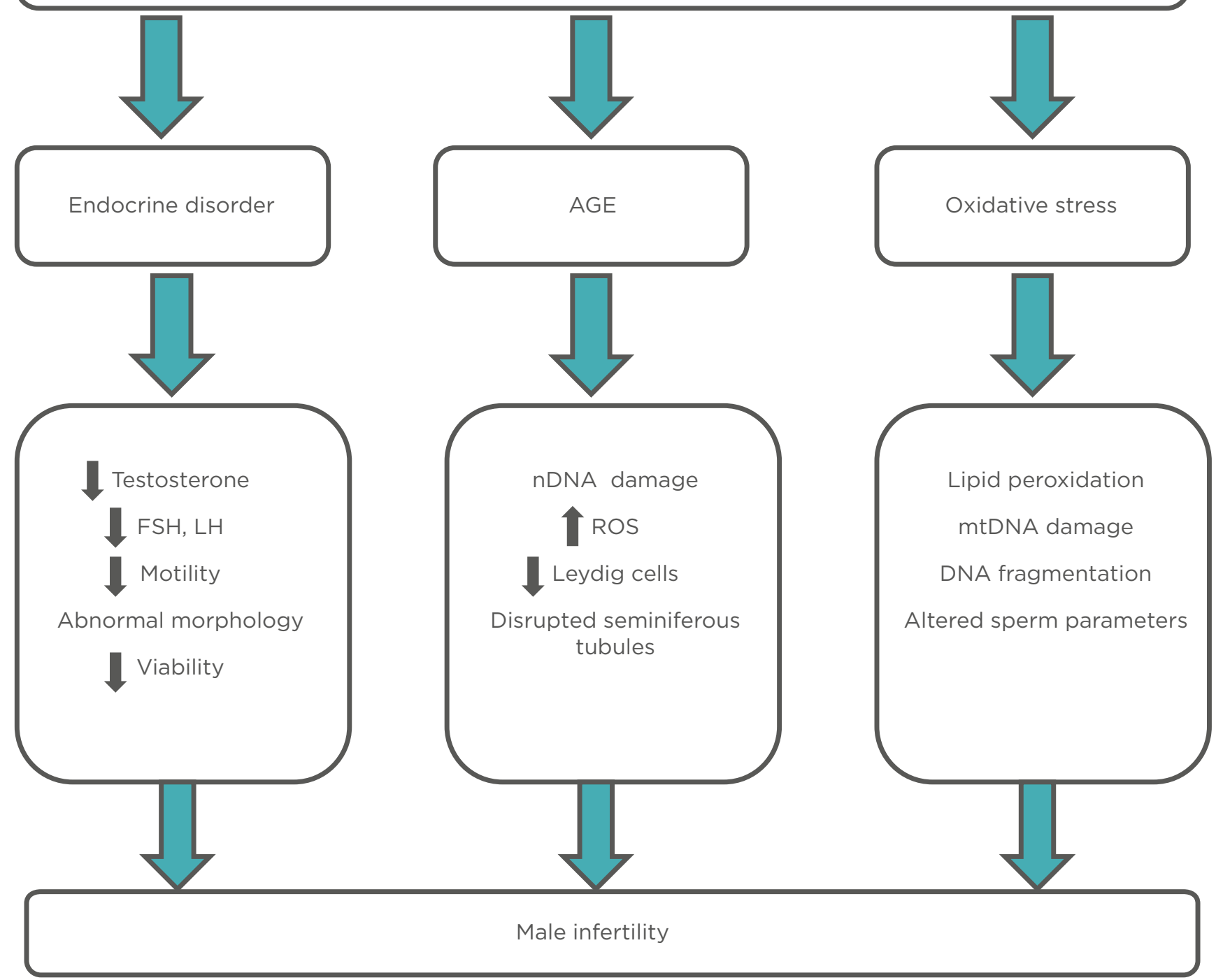

Figure 2: Mechanisms through which diabetes affects reproductive functions in males.

AGE: advanced glycation end products; FSH: follicle-stimulating hormone; LH: luteinizing hormone; mtDNA: mitochondria DNA; nDNA: nuclear DNA; ROS: reactive oxygen species.

Adapted from Temidayo et al. ${ }^{58}$

\section{References}

1. International Diabetes Federation (IDF). IDF Diabetes Atlas $8^{\text {th }}$ edition 2017. 2017. Available at: https:// diabetesatlas.org/en/. Last accessed: 30 May 2020.

2. Gandhi J et al. The role of diabetes mellitus in sexual and reproductive health: an overview of pathogenesis, evaluation, and management. Curr Diabetes Rev. 2017;13(6):573-81.
3. Tamás V, Kempler P. Sexual dysfunction in diabetes. Handb Clin Neurol. 2014;126:223-32

4. Hackett G. Metabolic effects of testosterone therapy in men with Type 2 diabetes and metabolic syndrome. Sex Med Rev. 2019;7(3):476-90.
5. Dhindsa S et al. Hypogonadotropic hypogonadism in men with diabesity. Diabetes Care. 2018;41(7):1516-25.

6. Bąk E et al. Relationships of sexual dysfunction with depression and acceptance of illness in women and men with Type 2 diabetes mellitus. Int J Environ Res Public Health 2017;14(9):1073. 
7. Dhindsa S et al. Frequent occurrence of hypogonadotropic hypogonadism in Type 2 diabetes. J Clin Endocrinol Metab. 2004;89(11):5462-8.

8. Kapoor D et al. Clinical and biochemical assessment of hypogonadism in men with Type 2 diabetes: correlations with bioavailable testosterone and visceral adiposity. Diabetes Care. 2007;30(4):911-7.

9. Grossmann M et al. Low testosterone levels are common and associated with insulin resistance in men with diabetes. J Clin Endocrinol Metab. 2008;93(5):1834-40.

10. Ho $\mathrm{CH}$ et al. The prevalence and the risk factors of testosterone deficiency in newly diagnosed and previously known Type 2 diabetic men. J Sex Med. 2015;12(2):389-97.

11. Chandel A et al. Testosterone concentration in young patients with diabetes. Diabetes Care. 2008;31(10):2013-7.

12. Dandona P, Dhindsa S. Update: Hypogonadotropic hypogonadism in Type 2 diabetes and obesity. J Clin Endocrinol Metab. 2011;96(9):2643-51.

13. Saboor Aftab SA et al. The role of obesity and Type 2 diabetes mellitus in the development of male obesityassociated secondary hypogonadism Clin Endocrinol (Oxf). 2013;78(3):330-7.

14. Dhindsa $S$ et al. Low estradiol concentrations in men with subnormal testosterone concentrations and Type 2 diabetes. Diabetes Care. 2011;34(8):1854-9.

15. Farooqi IS et al. Beneficial effects of leptin on obesity, T cell hyporesponsiveness, and neuroendocrine/metabolic dysfunction of human congenital leptin deficiency. J Clin Invest. 2002;110(8):1093-103.

16. Seminara SB et al. The GPR54 gene as a regulator of puberty. $\mathrm{N}$ Engl J Med. 2003;349(17):1614-27.

17. George JT et al. Exploring the pathophysiology of hypogonadism in men with Type 2 diabetes: kisspeptin-10 stimulates serum testosterone and $\mathrm{LH}$ secretion in men with Type 2 diabetes and mild biochemical hypogonadism. Clin Endocrinol (Oxf). 2013;79(1):100-4.

18. Watanobe H, Hayakawa Y. Hypothalamic interleukin-1 beta and tumor necrosis factor alpha, but not interleukin- 6 , mediate the endotoxin-induced suppression of the reproductive axis in rats. Endocrinology. 2003;144(11):4868-75.

19. Bhatia $\vee$ et al. Low testosterone and high C-reactive protein concentrations predict low hematocrit in Type 2 diabetes Diabetes Care. 2006;29(10):2289-94.

20. Dandona P et al. Inflammation: the link between insulin resistance, obesity and diabetes. Trends Immunol. 2004;25(1):4-7.

21. Dhindsa S et al. Insulin resistance and inflammation in hypogonadotropic hypogonadism and their reduction after testosterone replacement in men with Type 2 diabetes. Diabetes Care. 2016;39(1):82-91

22. Jones TH et al.; TIMES2 Investigators. Testosterone replacement in hypogonadal men with Type 2 diabetes and/or metabolic syndrome (the TIMES2 study). Diabetes Care. 2011;34(4):828-37.

23. Gianatti EJ et al. Effect of testosterone treatment on glucose metabolism in men with Type 2 diabetes: a randomized controlled trial. Diabetes Care. 2014;37(8):2098107.

24. Hackett G et al.; BLAST Study Group. Testosterone replacement therapy improves metabolic parameters in hypogonadal men with Type 2 diabetes but not in men with coexisting depression: the BLAST study. J Sex Med. 2014;11(3):840-56

25. Muraleedharan $\mathrm{V}$ et al. Testosterone deficiency is associated with increased risk of mortality and testosterone replacement improves survival in men with Type 2 diabetes. Eur J Endocrinol. 2013;169(6):725-33.

26. Farias JM et al. Low testosterone concentration and atherosclerotic disease markers in male patients with Type 2 diabetes. J Clin Endocrinol Metab. 2014;99(12):4698-703.

27. Daka B et al. Low concentrations of serum testosterone predict acute myocardial infarction in men with Type 2 diabetes mellitus. BMC Endocr Disord. 2015;15:35.

28. Muraleedharan $\mathrm{V}$, Jones $\mathrm{TH}$. Testosterone and mortality. Clin Endocrinol (Oxf). 2014;81(4):477-87.

29. Orwoll ES, Klein RF. Osteoporosis in men. Endocr Rev. 1995;16(1):87-116.

30. Khosla S et al. Relationship of serum sex steroid levels and bone turnover markers with bone mineral density in men and women: a key role for bioavailable estrogen. J Clin Endocrinol Metab. 1998;83(7):2266-74

31. Dhindsa S et al. The effects of hypogonadism on body composition and bone mineral density in Type 2 diabetic patients. Diabetes Care. 2007;30(7):1860-1.

32. Goodman $\mathrm{N}$ et al. American Association of Clinical Endocrinologists and American College of Endocrinology position statement on the association of testosterone and cardiovascular risk. Endocr Pract. 2015;21(9):1066-73.

33. Bhasin S et al. Testosterone therapy in men with hypogonadism: an Endocrine Society guideline. J Endocrinol Metab. 2018;103(5):1715-44.
34. Introduction: Standards of Medica Care in Diabetes - 2018. Diabetes Care. 2018;41(Suppl 1):S1-2.

35. Dhindsa S et al. Relationship of prostate-specific antigen to age and testosterone in men with Type 2 diabetes mellitus. Endocr Pract. 2008;14(8):1000-5.

36. Grossmann M. Low testosterone in men with Type 2 diabetes: significance and treatment. J Clin Endocrinol Metab. 2011;96(8):2341-53.

37. Corona $\mathrm{G}$ et al. Cardiovascular risk associated with testosteroneboosting medications: a systematic review and meta-analysis. Expert Opin Drug Saf. 2014;13(10):1327-51.

38. AbbVie. A Study to Evaluate the Effect of Testosterone Replacement Therapy (TRT) on the Incidence of Major Adverse Cardiovascular Events (MACE) and Efficacy Measures in Hypogonadal Men (TRAVERSE). NCT03518034. https://clinicaltrials. gov/ct2/show/NCT03518034.

39. Derosa G et al. Prevalence and associations of erectile dysfunction in a sample of Italian males with Type 2 diabetes. Diabetes Res Clin Pract. 2015;108(2):329-35.

40. Kouidrat $Y$ et al. High prevalence of erectile dysfunction in diabetes: a systematic review and metaanalysis of 145 studies. Diabet Med. 2017;34(9):1185-92

41. Angulo J et al. Diabetes exacerbates the functional deficiency of NO/ cGMP pathway associated with erectile dysfunction in human corpus cavernosum and penile arteries. J Sex Med. 2010;7(2 Pt 1):758-68.

42. Traish AM et al. Adipocyte accumulation in penile corpus cavernosum of the orchiectomized rabbit: a potential mechanism for veno-occlusive dysfunction in androgen deficiency. J Androl. 2005;26(2):242-8.

43. Chrysant SG. Antihypertensive therapy causes erectile dysfunction. Curr Opin Cardiol. 2015;30(4):383-90.

44. Arafa M et al. The prevalence of Peyronie's disease in diabetic patients with erectile dysfunction. Int J Impot Res. 2007;19(2):213-7.

45. Billups KL. Erectile dysfunction as an early sign of cardiovascular disease. Int J Impot Res. 2005;17(Suppl 1):S19-24.

46. Yamada $T$ et al. Erectile dysfunction and cardiovascular events in diabetic men: a meta-analysis of observational studies. PLoS ONE. 2012;7(9):e43673.

47. Rosen RC et al. Development and evaluation of an abridged, 5-item version of the International Index of Erectile Function (IIEF5) as a diagnostic tool for erectile dysfunction. Int J Impot Res. 1999;11(6):319-26. 
48. Hackett $\mathrm{G}$ et al. British Society for Sexual Medicine guidelines on the management of erectile dysfunction in men - 2017. J Sex Med. 2018;15(4):430-57.

49. Kostis JB, Dobrzynski JM. The effect of statins on erectile dysfunction: a meta-analysis of randomized trials. J Sex Med. 2014;11(7):1626-35.

50. Chen $Y$ et al. Losartan improves erectile dysfunction in diabetic patients: a clinical trial. Int J Impot Res. 2012;24(6):217-20.

51. Ramirez CE et al. Treatment with sildenafil improves insulin sensitivity in prediabetes: a randomized, controlled trial. J Clin Endocrinol Metab. 2015;100(12):4533-40.

52. Linet Ol, Ogrinc FG. Efficacy and safety of intracavernosal alprostadil in men with erectile dysfunction. The Alprostadil Study Group. N Engl J Med. 1996;334(14):873-7.

53. Fulgham PF et al. Disappointing initial results with transurethral alprostadil for erectile dysfunction in a urology practice setting. J Urol. 1998;160(1):2041-6.
54. Garber BB. Inflatable penile prosthesis: results of 150 cases. $\mathrm{Br} J$ Urol. 1996;78(6):933-5.

55. Rowland DL et al. The psychologica burden of premature ejaculation. J Urol. 2007;177(3):1065-70.

56. Arafa M, El Tabie O. Medica treatment of retrograde ejaculation in diabetic patients: a hope for spontaneous pregnancy. J Sex Med. 2008;5(1):194-8

57. World Health Organization (WHO). Global report on diabetes. 2016. Available at: https://www.who.int/ diabetes/global-report/en/. Last accessed: 10 September 2020

58. Temidayo SO, Stefan SP. Diabetes mellitus and male infertility. Asian Pacific J Reprod. 2017;7(1):6-14.

59. Bhattacharya SM et al. Diabetes mellitus and abnormalities in semen analysis. J Obstet Gynaecol Res. 2014;40(1):167-71.

60. Delfino $M$ et al. Prevalence of diabetes mellitus in male partners of infertile couples. Minerva Urol Nefrol. 2007:59(2):131-5
61. Dinulovic D, Radonjic G. Diabetes mellitus/male infertility. Arch Androl. 1990;25(3):277-93.

62. Baccetti B et al. Insulin-dependent diabetes in men is associated with hypothalamo-pituitary derangement and with impairment in semen quality. Hum Reprod. 2002;17(10):2673-7.

63. Karimi $\mathrm{J}$ et al. Increased receptor for advanced glycation end products in spermatozoa of diabetic men and its association with sperm nuclear DNA fragmentation. Andrologia. 2012;44(Suppl 1):280-6.

64. Maresch CC et al. Diabetes-induced hyperglycemia impairs male reproductive function: a systematic review. Hum Reprod Update. 2018;24(1):86-105.

65. Moss JL et al. Effect of rejuvenation hormones on spermatogenesis. Fertil Steril. 2013;99(7):1814-20.

66. Kessopoulou E et al. A double-blind randomized placebo cross-over controlled trial using the antioxidant vitamin $\mathrm{E}$ to treat reactive oxygen species associated male infertility. Fertil Steril. 1995;64(4):825-31. 